\title{
Estevia (stevia rebaudiana), edulcorante natural y no calórico
}

\author{
Stevia (stevia rebaudiana), \\ non-caloric natural sweetener
}

\begin{abstract}
Since ancient times mankind has had a marked preference for sweet foods. Sweeteners provide the same sensations as sugar. Stevia has been used for many years for a variety of purposes, both as a sweetener and medicine, especially in the management of diabetes by reducing plasma glucose levels and insulin, suggesting that Stevia could help in glucose regulation. It is a white crystalline compound (stevioside) that is a natural herbal sweetener without calories, and is between 100 to 300 times sweeter than sugar. The stevioside seems to have little or no acute toxicity. Its use as supplement is safe and does not stimulate appetite, so there is no risk of weight gain in its consumption. Key words: Stevia rebaudiana, no caloric sweetener, diabetes.
\end{abstract}

\section{INTRODUCCIÓN}

El consumo de bebidas azucaradas puede ser una de las causas dietarías de trastornos metabólicos como la obesidad. El sustituir el azúcar por edulcorantes bajos en calorías puede ser una estrategia eficaz de control de peso (1).

El consumo de azúcares agregados en los Estados Unidos se ha incrementado en casi un $20 \%$ en las últimas décadas, se estima que el consumo actual es de 142 libras por persona al año (equivalente a 64,4 kilos) (2). El consumo de alimentos y bebidas azucarados puede influir significativamente en el índice glicémico de cada comida así como la dieta en su conjunto. Por otra parte, la ingesta excesiva de calorías y alimentos de alto índice glicémico pueden dar lugar a una glucosa postprandial incrementada y los elevados niveles de insulina pueden conducir a cambios metabólicos y hormonales que estimulan la sensación de hambre y promueven la acumulación de grasa (3). Estudios realizados en esa línea sugieren que el consumo de bebidas endulzadas con azúcar promueven un balance energético positivo, por lo tanto un aumento de peso y el incremento en el riesgo de diabetes (4). Por otro lado, se ha encontrado que el consumo excesivo de fructosa causa dislipidemia, aumenta la adiposidad visceral y disminuye la sensibilidad a la insulina en sujetos
Samuel Durán A. (1)

María del Pilar Rodríguez N. (2)

Karla Cordón A. (3)

Jiniva Record C. (4)

(1) Nutrición y Alimentación, Carrera de Nutrición y Dietética. Universidad Autónoma de Chile, Santiago, Chile; (2) Nutrición y Alimentación. Carrera de Nutrición y Dietética. Universidad Santo Tomás, sede Viña del Mar, Chile;

(3) Andragogía y Educación Superior, Carrera de Nutrición. Facultad de Ciencias Químicas y Farmacia, Universidad de San Carlos de Guatemala, Guatemala; (4) Nutrición y Alimentación. Carrera de Nutrición y Dietética. Universidad Latinoamericana de Ciencia y Tecnología (ULACIT), Panamá.

Dirigir la correspondencia a: Profesor

Samuel Durán Agüero Universidad Autónoma de Chile Ricardo Morales 3369, San Miguel Santiago de Chile

E-mail: sduran74@gmail.com

Este trabajo fue recibido el 12 de Junio de 2012 y aceptado para ser publicado el 8 de Octubre de 2012.

con sobrepeso (5).

Una alternativa tanto al azúcar como a la fructosa es el uso de edulcorantes no nutritivos. El consumo de alimentos y bebidas que contienen edulcorantes no nutritivos ha aumentado dramáticamente en las últimas décadas, se estima que en Estados Unidos un 15\% de la población consume edulcorantes no nutritivos (6). En cambio un estudio realizado en escolares chilenos muestra que la ingesta es cercana al 99\% (7).

Entre los edulcorantes no nutritivos podemos mencionar la Sacarina, Aspartame, Sucralosa, Ciclamato, Acesulfame K, Neotamo, Alitamo y recientemente se ha incorporado la Estevia, cuyo sabor es lo más parecido al azúcar, distinguiéndose de los edulcorantes artificiales por no tener sabor metálico y no ser cancerígeno.

La Stevia rebaudiana es una planta originaria del Sudeste de Paraguay, miembro de la familia de las asteráceas, conocida como "hoja dulce". Es un arbusto perenne que puede alcanzar 65 a $80 \mathrm{~cm}$, pero que cultivadas pueden Ilegar hasta 1,0 m de altura, sus hojas lanceoladas tienen aproximadamente $5 \mathrm{~cm}$ de longitud y $2 \mathrm{~cm}$ de ancho y se disponen alternadas, enfrentadas de dos en dos. Puede utilizarse para la producción comercial por un periodo de cinco o más años, dando varias cosechas 
anuales a partir de la parte aérea de la planta, crece en suelos arenosos cerca de arroyos de la parte selvática subtropical del alto Paraná (8).

\section{PROPIEDADES DE LA ESTEVIA}

Durante siglos, las tribus Guaraníes de Paraguay y Brasil han usado diferentes especies de Estevia, principalmente Stevia rebaudiana, como endulzante para contrarrestar el sabor amargo de los medicamentos a base de diferentes plantas y bebidas, y con fines medicinales que incluyen la regulación de la glicemia e hipertensión (5). Este efecto hipotensor leve se observó en sujetos tratados con té de Stevia rebaudiana, administrado diariamente por 30 días (9). Se reporta como anticonceptivo (10), en el tratamiento de alteraciones de la piel y en prevención de caries, ya que no puede ser fermentado (11). Se ha informado que tiene efectos bactericidas sobre Streptococcus mutans, responsable de las caries dentales al poseer propiedades antibacterianas $(8,12)$ y antivirales $(13)$.

Además estimulan el estado de alerta, facilitan la digestión, las funciones gastrointestinales y mantiene la sensación de vitalidad y bienestar. La disminución del deseo de comer dulces y alimentos grasos es reportado por consumidores de Estevia. Otros en cambio indican que su consumo reduce el deseo del tabaco y de bebidas alcohólicas (14).

El botánico suizo Moisés Santiago Bertoni fue el primero que la describió en 1887, detallando su sabor dulce. En 1900, el químico paraguayo Ovidio Rebaudi, logró aislar los principios activos responsables del dulzor (10).

Estevia no contiene calorías y las hojas pueden utilizarse en su estado natural, gracias a su gran poder edulcorante, y sólo son necesarias pequeñas cantidades del producto $(11,15,16)$.

\section{Componentes de la Estevia}

En 1997 Ngowata purificó el extracto de Estevia obteniendo un Esteviósido, un polvo blanco y altamente higroscópico, por lo cual hay que mantenerlo en un envase hermético para evitar la humedad. En la producción a gran escala se utiliza el mismo método anterior, salvo para el paso final que genera productos secos mediante el uso de una secadora en aerosol. Los investigadores informaron que de $3000 \mathrm{~g}$ de Estevia se podía producir 101,5 g de polvo fino de color amarillo de Esteviósido.

Extractos de la Stevia rebaudiana se utilizan como edulcorante natural o en suplementos dietéticos por su contenido de glucósidos: Esteviósido y rebaudiósido con características químicas y farmacológicas adecuadas para su uso en la alimentación humana. Los principios de la Stevia rebaudiana se deben a sus componentes naturales activos presente en las hojas que son el Esteviósido y rebaudiosidos $A, B, C, D$ y E; Dulcósido A, y Esteviolbiósido. El Esteviósido tiene un ligero sabor amargo y proporciona 250 a 300 veces el dulzor del azúcar (17).

Las hojas de la planta silvestre de Estevia contienen 0,3\% Dulcósido, 0,6\% Rebaudiósido C, 3,8\% Rebaudiósido A y el 9,1\% de Esteviósido. La composición química completa de las especies de Estevia aún no está disponible. De las 110 especies estudiadas por el sabor dulce solo 18 muestran esta característica. De todas las especies la Stevia rebaudiana bertoni es la más dulce.

De acuerdo a Sharma y cols (2006) (18) las hojas frescas de Estevia contienen una gran cantidad de agua (80 a 85\%). Aparte de los componentes antes mencionados (glucósidos), las hojas contienen ácido ascórbico, $\beta$-caroteno, cromo, cobalto, magnesio, hierro, potasio, fósforo, riboflavina, tiamina, estaño, zinc, etc. Entre los productos químicos encontrados están la apigenina, austroinilina, avicularin, $\beta$-sitoesterol, ácido caféico, campesterol, cariofileno, centaureidin, ácido clorogénico, clorofila, kaempferol, luteolina, quercetina, estigmasterol, entre otras.

\section{Seguridad en su consumo}

El Esteviósido parece tener muy poca o ninguna toxicidad aguda $(19,20)$. Del mismo modo, el consumo crónico de Esteviósido se cree que representa poco riesgo basado en los estudios en humanos. Yamada en 1985 mostró que el consumo oral de Esteviósido en cantidades elevadas como $550 \mathrm{mg} / \mathrm{kg}$ de peso corporal al día (es decir 200 veces la ingesta máxima probable de alrededor de $2 \mathrm{mg} / \mathrm{kg} /$ peso corporal/día (21) por 2 años, no tuvo efectos tóxicos o cancerígenos en ratas. Sin embargo los efectos farmacológicos son sugeridos por otros estudios, como la reducción de la presión arterial y los niveles de glucosa en sangre. Además, el metabolito de la aglicona, el esteviol, se informó que es mutagénico y bactericida en Salmonella typhimurium TM677 (22). Por lo tanto, los efectos biológicos e interacciones adversas con fármacos se desconocen.

\section{Regulación y producción}

Ante la creciente demanda de productos bajos en calorías o sin calorías, Estevia ha tomado un sitio muy importante en la canasta familiar, se emplea como edulcorante de mesa, en la elaboración de bebidas, dulces, mermeladas, chicles, en pastelería, confituras, yogures, entre otros. Pero además de sus propiedades endulzantes, Estevia tiene importantes efectos sobre la salud (10).

Recientemente fue aprobado para su utilización comercial por el Joint Food and Agriculture Organization/World Health Organization Expert Committee on Food Additives (Joint Food and Agriculture Organization/World Health Expert Committee on Food Additives, 2005), y mas recientemente la aprobación como Generalmente Reconocido como Seguro (GRAS por sus siglas en ingles) de la Food and Drug Administration (23).

El Comité Mixto FAO/OMS de Expertos en Aditivos Alimentarios (JECFA) en sus reuniones $68^{\mathrm{a}}$ y $69^{\mathrm{a}}$ del año 2008 , estableció una Ingestión Diaria Admisible (IDA) para los glucósidos de Esteviol de 0-4 mg por kg de peso corporal por día, expresada como Esteviol (24). Los glucósidos de Esteviol son una mezcla de componentes de diferentes pesos moleculares. Dado que el componente activo efectivo es la parte de Esteviol de las distintas moléculas, la IDA se refiere al peso molecular del total de Esteviol presente en la mezcla (24).

A escala comercial la utilización de este edulcorante está de moda desde principios de los setenta en Japón. Actualmente se cultiva en Japón, Brasil, el sudeste asiático, Canadá y China, siendo este último el principal exportador de esteviósido. En Japón, el cultivo de Estevia se emplea principalmente como sustituto de azúcar. Cerca de un cuarto de cucharadita de hojas es equivalente a una cucharadita de azúcar.

\section{Estevia y diabetes mellitus}

Los extractos de esteviósido rebaudiana pueden disminuir el nivel de glucosa en sangre en ratas diabéticas con un efecto tiempo-dependiente (8), resultados similares a los obtenidos utilizando polvo de hojas de Estevia (25). Los Esteviósidos regulan el nivel de glucosa en la sangre por el incremento en la secreción de insulina y una mejor utilización de la glucosa por los tejidos periféricos y los músculos en ratas diabéticas (26); también se postula que los esteviósidos contrarrestan la glucotoxicidad en la células beta o también suprime la secreción 
de glucagón por parte de las células a del páncreas $(27,28)$.

Las ratas y los ratones muestran preferencias por algunos edulcorantes no calóricos (29). Aunque la sacarina ha sido útil en el estudio del sabor dulce, es un pobre sustituto del azúcar para las ratas, la sacarina en su concentración máxima es preferida de forma similar a la sacarosa diluida (30). Un reciente estudio muestra que Estevia es atractivo para las ratas, pero estimula en menor medida el consumo de bebidas que las endulzadas con sacarina (31).

En estudios en humanos (voluntarios sanos), se investigó el efecto de la administración de extractos de Stevia rebaudiana en las pruebas de tolerancia a glucosa. A los sujetos se les dio un extracto acuoso de hojas de $5 \mathrm{~g}$ cada 6 horas por 3 días y se realizó una prueba de tolerancia a la glucosa antes y después de la administración de los extractos. Los resultados mostraron que el tratamiento con Estevia aumentó la tolerancia a la glucosa y disminuyó las concentraciones de glucosa plasmática (32). Diversos autores concluyen que la planta podría tener un uso potencial en el manejo de la diabetes tipo 2 .

\section{Estevia y apetito}

Los edulcorantes no nutritivos han mostrado resultados contradictorios con respecto al consumo de energía y el peso corporal, especialmente aspartame. La mayoría de los estudios indica que aspartame reduce la ingesta de alimentos y puede ayudar a controlar el peso (33). Otros en cambio sugieren que el aspartame podría incrementar el apetito. Estudios que han evaluado el efecto de Estevia sobre el apetito indican que los sujetos que consumieron aspartame y Estevia no compensaron comiendo más en la siguiente comida (almuerzo o cena) y presentaron niveles similares de saciedad en comparación con los sujetos que consumieron sacarosa. Adicionalmente, Estevia redujo los niveles de glucosa plasmática e insulina, lo que sugiere que Estevia podría ayudar con la regulación de la glucosa (1).

\section{Absorción y excreción de Estevia}

La absorción de esteviósido, distribución y metabolismo se han evaluado en animales de experimentación.

El esteviósido puede ser degradado a esteviol, por la flora intestinal de diversas especies animales, incluido el hombre (34); el esteviósido es una molécula neutra con ambas regiones polares e hidrofóbicas, mientras que el esteviol contiene un anillo hidrofóbico y una carga negativa en el grupo carboxílico. En las ratas, el esteviósido no se absorbe con eficacia, en cambio el esteviol se absorbe rápidamente (34). De la misma forma, el esteviósido muestra una absorción muy limitada por las monocapas de las células Caco-2, en tanto que el esteviol se absorbe mucho mejor (35). Además, la secreción intestinal es limitada en esteviol. Como se ha sugerido anteriormente tras el consumo de esteviósido solo el esteviol se absorbe, además al parecer el esteviósido muestra una conversión metabólica casi por completo a esteviol en vivo. Por lo tanto la ingesta diaria admisible de $7,9 \mathrm{mg} / \mathrm{kg}$ de peso corporal/día de esteviósido K sugerido por Xili (21) produciría una concentración plasmática máxima de aproximadamente 0,2 nM de esteviol, asumiendo una conversión total de esteviósido a esteviol y la que la absorción es total en el intestino.

Se ha evaluado el manejo renal del esteviósido y del esteviol. Se ha informado de que el clearance de esteviósido fue mayor que la depuración de inulina, lo que indica que el esteviósido es secretado por el epitelio tubular renal (36). Estudios en ratas muestran que la administración oral de esteviol-17, da como resultado una excreción tanto en las heces como en la orina. Además al utilizar esteviol marcado radiactivo, se encuentra principalmente en la orina después de ligar el conducto biliar tras la administración oral o intracecal, lo que indica que el esteviol se absorbe en el intestino grueso y se excreta por los sistemas renal y biliar (37). A pesar de lo anterior, los mecanismos subyacentes de la excreción de esteviósido y esteviol son todavía desconocidos. Esteviósido y esteviol han demostrado que inducen la diuresis y natriuresis, sin un cambio significativo en la tasa de filtración glomerular o el flujo plasmático renal (38).

\section{CONCLUSIONES}

Estevia es un edulcorante no calórico, de origen natural, que se cultiva y utiliza en diversas partes del mundo y que ha penetrado de manera importante en el mercado nacional e internacional. Publicaciones reportan propiedades positivas entre ellas en el manejo de la diabetes mellitus transformándose en una nueva herramienta nutricional, Organismos internacionales avalan su consumo como suplemento seguro y no estimula el apetito por ende sin riesgo de incremento de peso en su consumo.

\section{RESUMEN}

Desde tiempos ancestrales la humanidad ha tenido una marcada preferencia hacia los alimentos dulces. Los edulcorantes proporcionan las mismas sensaciones que produce el azúcar, entre ellos estevia que se ha utilizado desde hace muchos años con diversos fines tanto como endulzante $y$ medicina, especialmente en el manejo de la diabetes, ya que reduce los niveles de glucosa plasmática e insulina, lo que sugiere que estevia podría ayudar con la regulación de la glucosa. Es un compuesto cristalino de color blanco (Esteviósido) endulzante natural sin calorías siendo 100 a 300 veces más dulce que el azúcar. El Esteviósido parece tener muy poca o ninguna toxicidad aguda, además su uso como suplemento es seguro y no estimula el apetito, por lo tanto no hay riesgo de incremento de peso en su consumo.

Palabras clave: Estevia rebaudiana, edulcorante no calórico, diabetes mellitus.

\section{BIBLIOGRAFIA}

1. Anton SD, Martin CK, Han H, Coulon S, Cefalu WT, Geiselman $P$, Williamson DA. Effects of stevia, aspartame, and sucrose on food intake, satiety, and postprandial glucose and insulin levels. Appetite. 2010;55: 37-43.

2. Wells HF, Buzby JC. Dietary Assessment of Major Trends in U.S. Food Consumption, 1970-2005. (Rep. No. 33) Washington DC: U.S. Department of Agriculture; 2008.

3. O'Keefe JH, Bell DS. Postprandial hyperglycemia/hyperlipidemia (postprandial dysmetabolism) is a cardiovascular risk factor. American J Cardiol 2007;100: 899-904.

4. Malik VS, Schulze MB, Hu FB. Intake of sugar-sweetened beverages and weight gain: a systematic review. Am J Clin Nutr 2006; 84:274-88.

5. Lee CN, Wong KL, Liu JC, Chen YJ, Cheng JT, Chan P. Inhibitory effect of stevioside on calcium influx to produce anti-hypertension. Planta Med 2001;67:796-9.

6. Mattes $R$, Popkin B. Nonnutritive sweetener consumption in humans: effects on appetite and food intake and their putative mechanisms. Am J Clin Nutr 2009; 89, 1-14.

7. Durán S, Quijada MP, Silva L, Almonacid N, Berlanga MR, Rodríguez MP. Niveles de ingesta diaria de edulcorantes no nutritivos en escolares de la región de Valparaíso. Rev Chil Nutr 2011; 38:444-9. 
8. Kujur RS, Singh V, Ram M, Yadava HN, Singh KK, Kumari S, Roy BK.Antidiabetic activity and phytochemical screening of crude extract of Stevia rebaudiana in alloxan-induced diabetic rats. Pharmacognosy Res $2010 ; 2: 258-63$.

9. Boeckh, EM. Pharmacological drial of a concentrated crude extract of Stevia rebaudiana Bertoni in healthy volunteers. Arquivos Biol Tecnol 1992; 35:299-314.

10. Melis MS. Effects of chronic administration of Stevia rebaudiana on fertility in rats. J Ethnopharmacol 1999; 67: 157-61.

11. Brandle JE, Richman A, Swanson AK, Chapman BP. Leaf ESTs from Stevia rebaudiana: a resource for gene discovery in diterpene synthesis. Plant Mol Biol 2002: 50:613-22.

12. Satishkumar J, Sarvanan MM, Seethalakshmi I. Invitro antimicrobial and antitumor activities of Stevia rebaudiana (Asteraceae) leaf extracts. Trop J Pharm Res 2008;7:1143-9.

13. Takahashi K, Matsuda M, Ohashi K, Taniquchi K, Nakaqomi $O, A b e Y$, et al. Analysis of anti-rotavirus activity of extract from Stevia rebaudiana. Antiviral Res 2001; 49:15-24.

14. Stevia rebaudiana Bertoni. Available from: http://www. emperorsherbiologist.com/stevia.php

15. Geuns JM. Stevioside. Phytochemistry 2003; 64: 913-21.

16. Totté N, Charon L, Rohmer M, Compernolle F, Baboeuf I, Geuns J. Biosynthesis of the diterpenoidsteviol, an entkaurene derivative from Stevia rebaudianaBertoni, via the methylerythritol phosphate pathway. Tetrahedron Letters 2000; 41:6407-10.

17. Kolb N, Herrera JL, Ferreyra DJ, Uliana RF. Analysis of sweet diterpene glycosides from Stevia rebaudiana: Improved HPLC method. I Agric Food Chem 2001; 49:4538-41.

18. Sharma $N$, Kaushal $N$, Chawla A, Mohan M, Sethi A, Sharma Y. Stevia rebaudiana-A review. Agrobios Newslett 2006. 5:46-8.

19. Matsui M, Matsui K, Kawasaki Y, Oda Y, Noguchi T, Kitagawa Y, Sawada M, Hayashi M, Nohmi T, Yoshihira K.Evaluation of the genotoxicity of stevioside and steviol using six in vitro and one in vivo mutagenicity assays. Mutagenesis. 1996; 11: 573-9.

20. Toskulkao C, Chaturat L, Temcharoen P, and Glinsukon $T$. Acute toxicity of stevioside, a natural sweetener and its metabolite, steviol, in several animal species. Drug Chem Toxicol 1997; 20: 31-44.

21. Xili L, Chengjiany B, Eryi $X$, Reiming $S$, Yuengming $W$, Haodong $S$, and Zhiyian H. Chronic oral toxicity and carcinogenicity study of stevioside in rats. Food ChemToxicol 1992; 30: 957-65.

22. Perzzuto JM, Compadre CM, Swanson SM, Nanayakkara $P D$, Kinghorn $A D$ Metabolically activated steviol, the aglycone of stevioside, is mutagenic. Proc Natl Acad Sci USA 1985; 82: 2478-82.

23. Expert Committee on Food Additives FAO/OMS. Summary and Conclusions of the 69th meeting of the Joint FAO/
WHO. FAO/OMS (online). 2008. (acceso 21 de febrero de 2010). Disponible en: http://www.fao.org/ag/agn/agns files/jecfa69_final.pdf.

24. Comité Mixto FAO/OMS de Expertos en Aditivos Alimentarios (JECFA). Hoja Informativa final 5.11.2008 Glucósidos de esteviol. FAO/OMS (online). 2008. (acceso 21 de febrero de 2010). Disponible en: http://www.fao.org/ag/ agn/agns/files/FACTSHEET_\%20STEVIOL\%20 GLYCOSIDES_final1.pdf.

25. Chang JC, Wu MC, Liu IM, Cheng JT. Increase of insulin sensitivity by stevioside in fructose-rich chow-fed rats. Hormone and Metabolic Geuns M J. Stevioside.Phytochemistry 2003; 64:913-21.

26. Chen TH, Chen SC, Chan P, Chu Y L, Yang HY, Cheng JT. Mechanism of the polyglycemic effect of stevioside, a glycoside of Stevia rebaudiana. Planta Med 2005; 71:108-13.

27. Shibata H, Sawa Y, Oka T, Sonoke S, Kim KK, Yoshioka M. Steviol and steviol glycoside. Glucosyltransferase activities in S. rebaudianaBertoni.Purification and partial characterization. Arch Biochem Biophys 1995; 321:390-6.

28. Chen J, Jeppesen PB, Nordentoft I, Hermansen K. Stevioside improves pancreatic? cell function during glucotoxicity via regulation of acetyl-CoA carboxylase. Am J Physiol Endocrinol Metab 2007;292:1906-16.

29. Bachmanov AA, Tordoff MG, Beauchamp GK. Sweetener preference of C57BL/6ByJ and 129P3/J mice. Chem Senses $2001 ; 26: 905-13$.

30. Smith JC, Sclafani A. Saccharin as a sugar surrogate revisited. Appetite 2002; 38:155-60.

31. Sclafani A, Bahrani M, Zukerman S, Ackroff K.Stevia and saccharin preferences in rats and mice. Chem Senses 2010;35:433-43.

32. Curi R, Alvarez M, Bazotte RB. 1986. Effect of Stevia rebaudiana on glucose tolerance in normal adult humans. Braz J Med Biol Res 1986; 19:771-4.

33. De la Hunty A, Gibson S, Ashwell M.A review of the effectiveness of aspartame in helping with weight control. British Nutr Found Nutr Bull 2006; 31:115-28.

34. Koyama E, Sakai N, Ohori Y, Kitazawa K, Izawa O, Kakegawa K, Fujino A, and Ui M. Absorption and metabolism of glycosidic sweeteners of stevia mixture and their aglycone, steviol, in rats and humans. Food Chem Toxicol 2003; 41: 875-83.

35. Geuns JM. Stevioside. Phytochemistry (Oxford) 2003; 64: 913-21.

36. Melis MS. Renal excretion of stevioside in rats. J Nat Prod 1992; 55: 688-90.

37. Wingard RE Jr, Brown JP, Enderlin FE, Dale JA, Hale RL, Seitz CT Intestinal degradation and absorption of the glycosidic sweeteners stevioside and rebaudioside A. Experientia (Basel) 1980; 36: 519-20.

38. Melis, MS.; Rocha, ST.; Augusto, A. Steviol effect, a glycoside of Stevia rebaudiana, on glucose clearances in rats. Braz J Biol 2009; 69: 371-4. 